\title{
A practical review of prognostic correlations of molecular biomarkers in glioblastoma
}

\author{
*Michael Karsy, MD, PhD, ${ }^{1}$ Jayson A. Neil, MD,1 Jian Guan, MD,1 Mark A. Mahan, MD,1 \\ Howard Colman, MD, PhD, ${ }^{1,2}$ and Randy L. Jensen, MD, $\mathrm{PhD}^{1,2}$ \\ ${ }^{1}$ Department of Neurosurgery, Clinical Neuroscience Center; and ${ }^{2}$ Huntsman Cancer Institute, University of Utah, Salt Lake City, \\ Utah
}

Despite extensive efforts in research and therapeutics, achieving longer survival for patients with glioblastoma (GBM) remains a formidable challenge. Furthermore, because of rapid advances in the scientific understanding of GBM, communication with patients regarding the explanations and implications of genetic and molecular markers can be difficult. Understanding the important biomarkers that play a role in GBM pathogenesis may also help clinicians in educating patients about prognosis, potential clinical trials, and monitoring response to treatments. This article aims to provide an up-to-date review that can be discussed with patients regarding common molecular markers, namely 0-6-methylguanine-DNA methyltransferase (MGMT), isocitrate dehydrogenase 1 and 2 (IDH1/2), p53, epidermal growth factor receptor (EGFR), platelet-derived growth factor receptor (PDGFR), phosphatase and tensin homolog (PTEN), phosphoinositide 3-kinase (PI3K), and 1p/19q. The importance of the distinction between a prognostic and a predictive biomarker as well as clinical trials regarding these markers and their relevance to clinical practice are discussed.

http://thejns.org/doi/abs/10.3171/2015.1.FOCUS14755

KEY WORDS GBM; biomarker; IDH1/2; MGMT; overall survival; progression-free survival

$\mathrm{G}$ Lioblastoma (GBM) is a WHO Grade IV tumor with a poor prognosis, significant comorbidity, and limited therapeutic options. It was originally classified as either primary, arising de novo, or secondary, arising from low-grade glioma. Mutations important to the classification of these tumors have been shown to be important in gliomagenesis. ${ }^{25}$ Some genes critical to the understanding of GBM tumorigenesis and prognosis include O-6-methylguanine-DNA-methyltransferase (MGMT), isocitrate dehydrogenase gene 1 and 2 (IDH1/2), p53, epidermal growth factor receptor $(E G F R)$, platelet-derived growth factor receptor (PDGFR), phosphatase and tensin homolog (PTEN), phosphoinositide 3-kinase (PI3K), and $1 \mathrm{p} / 19 \mathrm{q}$ (Fig. 1 and Table 1). These genes serve as biomarkers of disease aggressiveness, provide insight into the pathophysiology of the disease, and may serve as potential sites for targeted treatment. In this review we aimed to evaluate the clinical impact of these gene markers as assessed on pathological tissue and how they impact prog- nosis as well as clinical decision making for the everyday clinician.

Recent interest in personalized medicine has spurred an investigation into individualized disease treatment through biomarker stratification. ${ }^{37}$ The US Food and Drug Administration defines a valid biomarker as "a biomarker that is measured in an analytical test system with well-established performance characteristics and for which there is an established scientific framework or body of evidence that elucidates the physiologic, toxicologic, pharmacologic, or clinical significance of test results." Biomarkers in GBM have been investigated for their use in stratifying prognosis, guiding the development of targeted treatment, and attempting to personalize clinical treatment. More specifically, prognostic biomarkers provide information on the natural history of the disease, and tests can be designed to distinguish disease recurrence in marker-positive and marker-negative patients regardless of clinical treatment. Alternatively, predictive markers are useful in identifying

ABBREVIATIONS $\mathrm{AA}=$ anaplastic astrocytoma; $\mathrm{GBM}=$ glioblastoma; $M G M T m=M G M T$ promoter methylation; $\mathrm{OS}=$ overall survival; $\mathrm{PFS}=$ progression-free survival. SUBMITTED November 1, 2014. ACCEPTED January 8, 2015. INCLUDE WHEN CITING DOI: 10.3171/2015.1.FOCUS14755.

DISCLOSURE The authors report no conflict of interest concerning the materials or methods used in this study or the findings specified in this paper. Dr. Jensen is a consultant for Medtronic, IMRIS, Pharmokokinesis, and Varian. Dr. Colman is a consultant for Hoffman La Roche, Genentech, Sigma Tau, Proximagen, and Foundation Medicine.

* Drs. Karsy and Neil contributed equally to this work. 


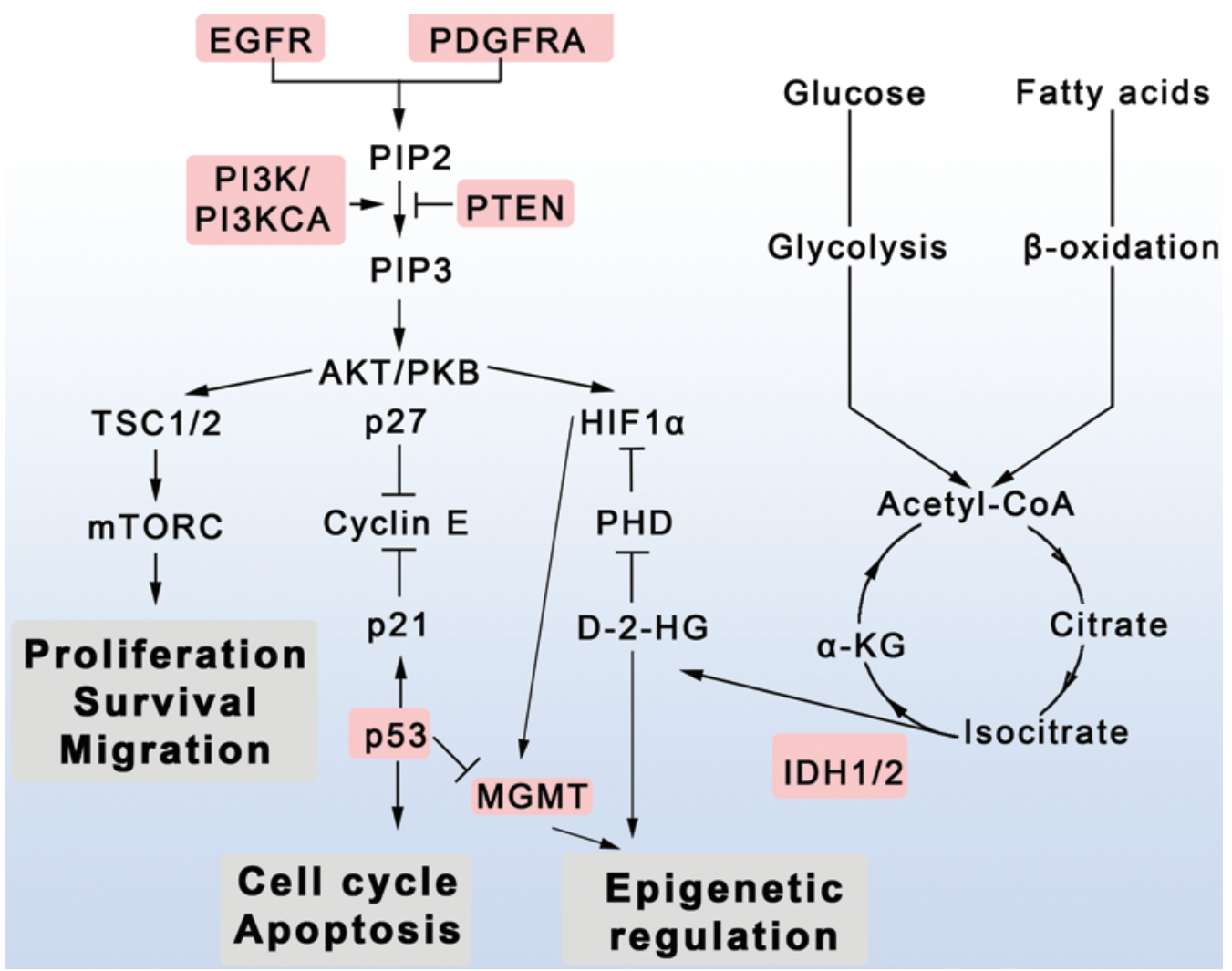

FIG. 1. Key pathways and biomarkers in GBM pathogenesis. The relationship between key biomarkers (red) and other signaling molecules are shown in GBM. $\alpha$-KG $=\alpha$-ketoglutarate; D-2-HG = D-2-hydroxyglutarate; HIF1 $\alpha=$ hypoxia-inducible factor-1 alpha; mTORC = mammalian target of rapamycin complex; PDGFRA = platelet-derived growth factor receptor $\alpha ;$ PHD = prolyl hydroxylase domain enzymes; PIP2 = phosphatidylinositol-4,5-bisphosphate; PIP3 = phosphatidylinositol-3,4,5-trisphosphate; TSC1/2 = tuberous sclerosis protein 1 and 2.

patients who may achieve a particular outcome based on a particular treatment. A predictive marker can help in the selection of patients who may be sensitive or resistant to a particular treatment, while prognostic markers can help inform patients about prognosis stratification. Several of the markers discussed in this review, including IDHI/2 and MGMT, have distinct roles as both prognostic and predictive biomarkers, which will be discussed.

The advent and spread of genomic screening technologies has expanded investigation into cancer as well as broadened the category of biomarkers. Recent investigations have added to the complexity of GBM treatment and counseling by describing 4 distinct molecular subtypes. These include the classic, mesenchymal, proneural, and neural types, which have distinct alterations in EGFR, neurofibromin 1 (NF1), PDGF, and uncharacterized gene pathways, respectively. ${ }^{20}$ The molecular fingerprint of individual GBM tumors has been associated with prognosis and treatment response. For example, further investigation has supported the association of younger patients, secondary GBM, and longer survival within the proneural subtype. In addition, the classic and proneural subtypes have shown improved prognosis with treatment, while the mesenchymal subtype correlates with a worse prognosis. However, while these findings have been an exciting avenue for research, the use of genomic data for everyday clinical prognosis and treatment remains limited. Genom- ic classification of tumors is not yet clinically feasible, nor do we have a strong mechanistic understanding of how these multiple genomic alterations affect clinical prognosis.

\section{0-6-Methylguanine-DNA-Methyltransferase (MGMT)}

$M G M T$ encodes a repair protein that removes alkylation at the $\mathrm{O} 6$ position of guanine, a common site altered by alkylating chemotherapy. Temozolomide commonly alkylates DNA at the N7 or O6 position of guanine to disrupt DNA replication and trigger cell death. Hypermethylation of this gene promoter (MGMTm) results in reduced $M G M T$ expression and thus an impaired ability for cells to repair from damage induced by chemotherapeutic agents and radiation. In the original trial by Stupp et al. evaluating temozolomide, MGMTm significantly impacted response to temozolomide with radiotherapy. ${ }^{17,46}$ Of the 206 cases available, $45 \%$ showed MGMTm and resulted in an independently favorable prognostic factor with a median overall survival (OS) of 21.7 months after radiochemotherapy treatment compared with 15.3 months for the nonmethylated MGMT samples that received treatment.

More recent randomized trials have supported the prognostic and predictive roles of MGMTm. In the NOA-04 randomized clinical trial of anaplastic astrocytoma (AA; 


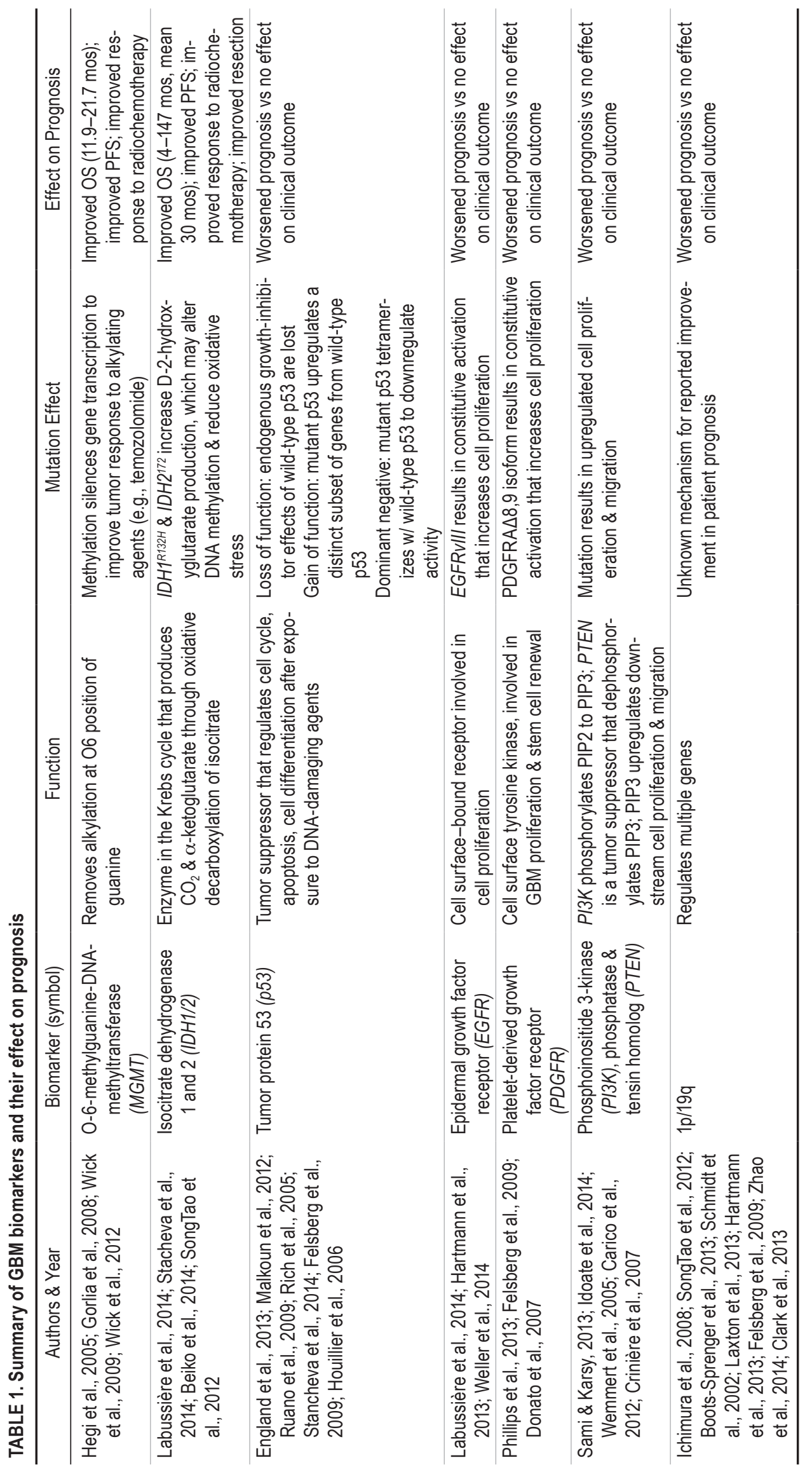


WHO Grade III), MGMTm was effective in predicting longer OS (11.9 vs 8.2 months) and progression-free survival (PFS; 8.4 vs 4.6 months), as well as improved response to chemotherapy (procarbazine, lomustine, and vincristine or temozolomide) compared with non-MGMTm controls.52 Interestingly, in patients who received only radiotherapy, a longer PFS was seen without MGMTm (3.3 vs 4.6 months), but the reason for this counter-intuitive effect is unclear. In the NOA-08 trial, 584 patients with GBM or AA were recruited for treatment with temozolomide alone or with radiotherapy ${ }^{53}$ MGMTm was seen in 73 of 209 tested patients (35\% of samples) and was associated with significantly longer OS (11.9 vs 8.2 months). Furthermore, event-free survival, defined as survival from surgery to first disease progression or death, was greater in patients with MGMTm who had chemotherapy than in non-MGMTm patients (8.4 vs 4.6 months). This result suggests that patients with MGMTm showed a greater response to chemotherapy; however, $M G M T$ status was not distinguished between patients with GBM and those with AA, limiting interpretation of the study. The European Organisation for Research and Treatment of Cancer (EORTC) 26981/22981 National Cancer Institute of Canada (NCIC) trial showed that $M G$ MTm also predicted improved responsiveness to temozolomide. ${ }^{15}$ Researchers in this study evaluated 573 patients with GBM who had been assigned to radiotherapy with or without temozolomide. Extent of tumor resection, younger patient age, Mini-Mental Status Examination score $>27$, and lack of corticosteroid treatment at baseline were all prognostic of better outcomes. The MGMTm status was identified in 96 of 206 samples $(47 \%$; 367 samples did not have MGMT results) in which $M G M T m$ was associated with an improved OS (HR 2.10, 95\% CI 1.54-2.85). Furthermore, response to radiochemotherapy was improved with MGMTm, although this result was not statistically significant. While this study lacked complete evaluation of MGMT status in all patients, a useful predictive normogram was presented and could be used for everyday clinical activity (www.eortc.be/tools/gbmcalculator). Some have advocated the use of MGMTm as a standard marker for GBM prognosis as well as to identify patients for clinical trials in evaluating alkylating therapies and/or radiation therapy.

\section{Isocitrate Dehydrogenase 1 and 2 (IDH1/2)}

IDHI/2 encode an enzyme in the Krebs cycle that produces $\mathrm{CO}_{2}$ and $\alpha$-ketoglutarate through oxidative decarboxylation of isocitrate. IDHI encodes a cytosolic protein, while $I D H 2$ encodes a mitochondrial protein. Identified in 2008 after a collaborative genomics study by Parsons et al., ${ }^{36} \mathrm{IDH} 1 / 2$ was shown to be mutated in approximately $5 \%$ of primary gliomas and $60 \%-80 \%$ of secondary gliomas. In fact, $I D H 1 / 2$ mutation may serve as an early driving mutation of GBM ${ }^{49} I D H 1^{R l 32 H}$ and $I D H 2^{172}$ mutations are the most common types of IDH mutations seen in GBM (> 90\% samples with IDHI/2 mutation) and result in increased production of the oncometabolite D-2-hydroxyglutarate, which may alter DNA methylation patterns in GBM and alter gene transcription on a wide number of targets. ${ }^{33} \mathrm{IDHI} / 2$ use nicotinamide adenine dinucleotide phosphate (NADP+) as a cofactor in NADPH production. Thus, mutations may decrease NADPH formation, resulting in increased oxidative stress, DNA oxidation, overwhelming of DNA repair mechanisms, and eventual induction of DNA damage. ${ }^{28}$

Multiple studies have validated both the prognostic and predictive benefit of IDHI/2 mutation. IDHI/2 mutations have been shown to improve OS from 4 months to as much as 147 months (mean 30 months), depending on the study. ${ }^{1,29,44,45}$ A recent study of 395 GBM samples showed $I D H 1 / 2$ mutations in 30 samples were associated with a significant improvement in OS (26.6 vs 14.5 months). ${ }^{29}$ Another recent study of 106 GBM samples, with 14 samples containing mutated $I D H I / 2$, also showed a significant improvement in OS (30.9 vs 7.7 months). ${ }^{45}$ Currently, multiple clinical trials are evaluating the use of $I D H 1 / 2$ as biomarkers for stratifying patients during targeted therapy in GBM (clinicaltrials.gov).

Several studies have shown a predictive effect for IDHI/2 in treatment efficacy. In a study of 88 cases of secondary GBM, IDH1/2 mutations correlated with improved PFS as well as response to temozolomide. ${ }^{44}$ An interesting study of 355 malignant astrocytomas (128 WHO Grade III, 207 WHO Grade IV) showed that an IDHI mutation correlated with improved MRI-defined enhancing disease and greater gross-total resection (93\% vs 67\%) and longer median OS (163.4 vs 16.2 months). ${ }^{1}$ Several clinical factors correlated with $I D H 1$ mutation, which may have improved resection, including younger patient age and frontal location of tumors; however, additional survival benefit (median survival 9.75 years) was gained from greater tumor resection $\left(<5 \mathrm{~cm}^{3}\right.$ residual) in $I D H I$ mutants but not $I D H I$ wild type. In addition to improved overall patient prognosis, IDH1/2 mutation results support enhanced therapeutic efficacy with chemoradiotherapy and greater resection.

\section{p53}

The $\mathrm{p} 53$ protein is encoded by the TP53 tumor suppressor gene, which is involved in regulation of the cell cycle, apoptosis, cell differentiation, and other mechanisms of cell regulation during exposure to DNA-damaging agents (for example, ultraviolet radiation, toxins). This gene is the most common mutation in GBM, found in $28 \%$ of primary GBMs and $65 \%$ of secondary GBMs. ${ }^{34}$ Three patterns of mutation occur with p53 dysfunction, namely loss of function, where endogenous growth-inhibitor effects of wild-type p53 are lost; gain of function, where mutant p53 upregulates a distinct subset of genes from wild-type p53; and dominant-negative effects, where mutant p53 tetramerizes with wild-type p53 to downregulate activity. ${ }^{12}$ Other mechanisms of p53 inactivation include mutations of its modulators including inhibitor MDM2 or deletion of p14 ${ }^{\text {ARF }}{ }^{2,32}$ Results from The Cancer Genome Atlas show that alterations in the p53 pathway (ARF/MDM2/MDM4/ p53) are found in $78 \%$ of GBMs, ${ }^{7}$ making it a common and important mutation in GBM.

Some studies support an effect of p53 status on improving prognosis, $, 31,39,40$ while others show a limited impact on clinical outcome. ${ }^{13,19,45}$ One study of 46 patients who had received adjuvant radiochemotherapy showed im- 
proved median PFS (9.3 vs 7 months) in samples without p53 overexpression; however, another study of $220 \mathrm{GBMs}$ did not show a significant difference in median survival between wild-type and mutant p53 GBM tumors (17.0 vs 14.7 months). ${ }^{19}$ Mutant p53 has been shown to induce radiochemotherapy resistance in many cancer types including GBM, ${ }^{24}$ with altered $\mathrm{p} 53$ expression resulting in increased sensitivity to temozolomide in one experimental model. ${ }^{3}$ Reasons for the lack of a clear correlation of p53 with prognosis are many-fold, including the complexity of the p53 signaling pathway, the importance of other regulators in the p53 pathway that may be altered in GBM (for example, Rb, MDM2), and the heterogeneity of p53 mutation types and effects. ${ }^{12}$ In fact, methods to target p53 in clinical trials have also been limited by these issues. ${ }^{12}$

\section{Epidermal Growth Factor Receptor (EGFR)}

$E G F R$ encodes a cell surface-bound receptor involved in cell proliferation with potential impacts on the clinical prognosis of GBM. Approximately 50\% of primary GBMs and $<10 \%$ of secondary GBMs show EGFR mutations. ${ }^{54}$ Furthermore, $10 \%-60 \%$ of primary GBMs contain the EGFR variant III mutation (EGFRvIII), with a deletion of the regulator N-terminal domain $(\Delta 6-273)$, which results in constitutive upregulation of mitogenic signaling pathways. ${ }^{14}$ Other mutation types of EGFR (for example, the C-terminal domain [C]-958, intergenic deletions [ $\Delta 521-603]$, duplication-insertion mutations [664-1030 and 664-1014], and others) exist but with unclear clinical significance. EGFRvIII can be detected in the peripheral blood of brain tumor patients, opening the possibility of screening patients for anti-EGFRvIII therapies and monitoring response. ${ }^{41}$

Several studies suggest the potential of EGFR as a prognostic factor in GBM given the presence of a specific EGFRvIII mutational variant with a clear role in gliomagenesis, ${ }^{29}$ while others have not shown a clinical correlation between EGFR and survival. ${ }^{16}$ In one study, EGFR amplification resulted in significantly lower OS than did EGFR wild type (13.3 vs 26.6 months) ${ }^{29}$ however, a study of long-term survivors of GBM (> 36 months) showed that alterations in $E G F R$ were not significant predictors. ${ }^{16}$ In a study from the German Glioma Network of 184 patients with GBM, EGFRvIII was seen in $18 \%$ of samples and did not show an effect on OS among mutant-containing and mutant-absent cases (9.6 vs 11.2 months). ${ }^{50}$ Moreover, EGFR amplification did not show an impact on OS compared with nonamplified samples (8.3 vs 6.4 months). Several current clinical trials in GBM aim to use EGFRvIII mutation to select patients for treatments, as was done for erlotinib in non-small cell lung cancer, as well as RNAdirected treatments and vaccine therapies. ${ }^{47}$ The goal of these trials is to eliminate the significant molecular variation in GBM tumor samples while using a specific targeted therapy. However, our limited understanding of how EGFR mutation changes downstream signaling pathways, such as AKT, MAPK, and STAT3, as well as differences in mutational types evaluated among various studies, complicates the clinical utility of this biomarker as well as its use for targeted treatment.

\section{Platelet-Derived Growth Factor Receptor (PDGFR)}

$P D G F R$ encodes a cell-surface tyrosine kinase similar to $E G F R$, which is also involved in GBM proliferation and stem cell renewal. PDGFR exists as multiple isoforms, with the $\alpha$ subtype (PDGFRA) playing the most significant role in GBM. PDGFR is mutated in up to $30 \%$ of GBMs,${ }^{5}$ and the PDGFRA $\Delta 8,9$ isoform (with a deletion of exons 8 and 9) results in constitutive activation and is seen in $40 \%$ of GBMs ${ }^{35}$ In a recent study, PDGFRA amplification was seen in $23 \%$ of GBM cases and demonstrated a significant reduction in median survival in only the IDH1 mutation subgroup (16.0 vs 72.6 months). ${ }^{38}$ Despite the importance of $P D G F$ in GBM proliferation-as it is found upstream of important signaling pathways such as AKT, involved with in vitro growth of GBM, and used in animal models of GBM-no definitive role in predicting prognosis has been seen in clinical studies. In one study of 65 cases of GBM, PDGFRA amplification was detected in only 1 case. $^{13}$ In another study of 43 cases of GBM, $P D G F R$ overexpression was not associated with survival. ${ }^{11}$ Results from The Cancer Genome Atlas suggest that PDGFRA alteration plays a critical role in the proneural subtype of GBM, but no alteration in prognosis was seen among evaluated samples. ${ }^{48}$

\section{Phosphatase and Tensin Homolog (PTEN) and Phosphoinositide 3-Kinase (PI3K)}

The PI3K/AKT pathway plays a critical role in regulating cell proliferation, migration, and other functions. ${ }^{42}$ PI3K phosphorylates phosphatidylinositol-4,5-bisphosphate (PIP2) to phosphatidylinositol-3,4,5-trisphosphate (PIP3) at the cell inner membrane, resulting in recruitment and upregulation of various downstream pathways including AKT. PTEN is the second most common tumor suppressor mutation after $p 53$ in GBM, where PTEN protein dephosphorylates PIP3 to PIP2 to suppress the downstream AKT pathway. Mutations of PTEN result in AKT upregulation and play a critical role in cell cycle regulation, apoptosis, and cell migration. Glioblastoma shows PTEN mutation or epigenetic silencing in $40 \%$ of cases, and loss of heterozygosity of chromosome 10 (LOH 10q23), where PTEN is located, is seen in $50 \%-90 \%$ of primary GBMs and $50 \%-70 \%$ of secondary GBMs. ${ }^{27}$ Furthermore, mutations in the catalytic p1 $10 \alpha$ subunit of PI3K (PIK3CA) are another mechanism of AKT upregulation but are seen in only $5 \%-13 \%$ of GBMs. ${ }^{26}$ PTEN has been shown in combination with $p 53$ mutation to form tumors resembling human gliomas in mouse knockout models. ${ }^{56}$

Despite the importance of PTEN in gliomagenesis, a distinct correlation with survival remains limited. Loss of heterozygosity of $10 \mathrm{q} 23$, where PTEN resides, has been shown to correlate with OS in some studies ${ }^{23,51}$ but not others. ${ }^{8,10}$ These findings suggest additional mechanisms govern PTEN signaling. Nevertheless, targeted treatments toward this pathway continue to be an active area of investigation. ${ }^{42}$

\section{$1 p / 19 q$}

Codeletion of chromosomes $1 \mathrm{p}$ and $19 \mathrm{q}$ can result in 
improved prognosis for oligodendrogliomas and has been explored in GBM. Deletion of 1p/19q improves PFS, OS, and response to chemotherapy and radiation in oligodendroglioma. ${ }^{6}$ Of note, a partial loss of $1 \mathrm{p}$ has been associated with a worse prognosis. Results from the Radiation Therapy Oncology Group (RTOG) 9402 and EORTC 26951 trials showed an improvement in OS with the addition of radiation to procarbazine/lomustine/vincristine chemotherapy in anaplastic oligodendroglioma with the $1 \mathrm{p} / 19 \mathrm{q}$ mutation. ${ }^{6}$ In GBM, similar findings have been demonstrated in some studies ${ }^{4,21,43,44}$ but not in others. ${ }^{13,16,30}$ In several studies, codeletion of $1 \mathrm{p}$ and $19 \mathrm{q}$ is associated with IDH1 mutation and MGMT hypermethylation., ${ }^{4,44}$ Nevertheless, in a study of 491 gliomas, 28 patients with GBM showed codeletion of $1 \mathrm{p} / 19 \mathrm{q}$, which did not change OS. ${ }^{9}$ Interestingly, $1 \mathrm{p} / 19 \mathrm{q}$ mutation was independent of $I D H I$ mutation, $10 \mathrm{q}$ loss, and EGFR alterations. A meta-analysis of 28 studies evaluating 3408 cases of glioma showed that $1 \mathrm{p} / 19 \mathrm{q}$ codeletion correlated with improved PFS (HR 0.63, 95\% CI $0.52-0.76$ ) and OS (HR $0.43,95 \%$ CI $0.35-0.53$ ) regardless of the WHO grade..$^{55}$ These results support further investigation into the effect of $1 \mathrm{p} / 19 \mathrm{q}$ mutation and whether the mechanism accounting for improved prognosis in some tumors exists and plays a role in GBM.

\section{Conclusions}

Despite the immense pace of research to understand the molecular basis of GBM, the clinical utility of key genes and markers remains limited. Further prospective research is needed to investigate the role of biomarkers and their effect on outcomes in diverse patient populations so that such markers will be better accepted clinically. Standardized assays for evaluating markers and treatments in prospective clinical trials are also warranted for a better understanding of how biomarkers may play a role in GBM. A recent survey of neurooncologists evaluated the use of biomarkers in GBM..$^{18}$ In the survey, MGMT (37\%), EGFR (23\%), 1p/19q (22\%)\%, EGFR (22\%), p53 (20\%), PTEN mutation or deletion (17\%), EGFRvIII (12\%), IDHI/2 (12\%), PDGFR (5\%), and PIK3CA (1\%) were the most common markers for which evaluation was requested for GBM specimens. Furthermore, $11 \%$ of respondents stated that knowing the MGMTm status was "always" or "almost always" helpful in clinical decision making versus $26 \%$ who reported it was "never" or "almost never" helpful. Authors of the survey concluded that more focused testing of biomarkers should be performed to improve patient care and reduce cost. Current evidence supports a role for MGMTm and $I D H I / 2$ mutation in predicting a mortality benefit as well as a benefit from current treatment modalities. The role of $p 53, P T E N, P I 3 K$, and $1 \mathrm{p} / 19 \mathrm{q}$ in prognostication remains limited probably because of the complexity of these signaling molecules as well as the diversity of GBM cases and clinical presentations. Glioblastoma remains a heterogeneous disease with individualized treatments, limited therapeutic options, and biomarkers to follow, as well as gaps in our understanding of its pathogenesis, but the use of biomarkers may allow better stratification of patients for clinical trials and treatment paradigms.

\section{Acknowledgments}

We thank Kristin Kraus, MSc, and Walavan Sivakumar, MD, for their editorial assistance in the preparation of this manuscript.

\section{References}

1. Beiko J, Suki D, Hess KR, Fox BD, Cheung V, Cabral M, et al: IDH1 mutant malignant astrocytomas are more amenable to surgical resection and have a survival benefit associated with maximal surgical resection. Neuro Oncol 16:81-91, 2014

2. Biernat W, Kleihues P, Yonekawa Y, Ohgaki H: Amplification and overexpression of MDM2 in primary (de novo) glioblastomas. J Neuropathol Exp Neurol 56:180-185, 1997

3. Blough MD, Beauchamp DC, Westgate MR, Kelly JJ, Cairncross JG: Effect of aberrant p53 function on temozolomide sensitivity of glioma cell lines and brain tumor initiating cells from glioblastoma. J Neurooncol 102:1-7, 2011

4. Boots-Sprenger SHE, Sijben A, Rijntjes J, Tops BBJ, Idema AJ, Rivera AL, et al: Significance of complete 1p/19q codeletion, IDH1 mutation and MGMT promoter methylation in gliomas: use with caution. Mod Pathol 26:922-929, 2013

5. Brennan C, Momota H, Hambardzumyan D, Ozawa T, Tandon A, Pedraza A, et al: Glioblastoma subclasses can be defined by activity among signal transduction pathways and associated genomic alterations. PLoS ONE 4:e7752, 2009

6. Cairncross G, Wang M, Shaw E, Jenkins R, Brachman D, Buckner J, et al: Phase III trial of chemoradiotherapy for anaplastic oligodendroglioma: long-term results of RTOG 9402. J Clin Oncol 31:337-343, 2013

7. Cancer Genome Atlas Research Network: Comprehensive genomic characterization defines human glioblastoma genes and core pathways. Nature 455:1061-1068, 2008

8. Carico C, Nuño M, Mukherjee D, Elramsisy A, Dantis J, Hu $\mathrm{J}$, et al: Loss of PTEN is not associated with poor survival in newly diagnosed glioblastoma patients of the temozolomide era. PLoS ONE 7:e33684, 2012

9. Clark KH, Villano JL, Nikiforova MN, Hamilton RL, Horbinski C: 1p/19q testing has no significance in the workup of glioblastomas. Neuropathol Appl Neurobiol 39:706-717, 2013

10. Crinière E, Kaloshi G, Laigle-Donadey F, Lejeune J, Auger $\mathrm{N}$, Benouaich-Amiel A, et al: MGMT prognostic impact on glioblastoma is dependent on therapeutic modalities. J Neurooncol 83:173-179, 2007

11. Donato V, Papaleo A, Castrichino A, Banelli E, Giangaspero F, Salvati M, et al: Prognostic implication of clinical and pathologic features in patients with glioblastoma multiforme treated with concomitant radiation plus temozolomide. Tumori 93:248-256, 2007

12. England B, Huang T, Karsy M: Current understanding of the role and targeting of tumor suppressor p53 in glioblastoma multiforme. Tumour Biol 34:2063-2074, 2013

13. Felsberg J, Rapp M, Loeser S, Fimmers R, Stummer W, Goeppert M, et al: Prognostic significance of molecular markers and extent of resection in primary glioblastoma patients. Clin Cancer Res 15:6683-6693, 2009

14. Frederick L, Wang XY, Eley G, James CD: Diversity and frequency of epidermal growth factor receptor mutations in human glioblastomas. Cancer Res 60:1383-1387, 2000

15. Gorlia T, van den Bent MJ, Hegi ME, Mirimanoff RO, Weller M, Cairncross JG, et al: Nomograms for predicting survival of patients with newly diagnosed glioblastoma: prognostic factor analysis of EORTC and NCIC trial 26981-22981/CE.3. Lancet Oncol 9:29-38, 2008

16. Hartmann C, Hentschel B, Simon M, Westphal M, Schackert $\mathrm{G}$, Tonn JC, et al: Long-term survival in primary glioblastoma with versus without isocitrate dehydrogenase mutations. Clin Cancer Res 19:5146-5157, 2013 
17. Hegi ME, Diserens AC, Gorlia T, Hamou MF, de Tribolet N, Weller M, et al: MGMT gene silencing and benefit from temozolomide in glioblastoma. N Engl J Med 352:997-1003, 2005

18. Holdhoff M, Ye X, Blakeley JO, Blair L, Burger PC, Grossman SA, et al: Use of personalized molecular biomarkers in the clinical care of adults with glioblastomas. J Neurooncol 110:279-285, 2012

19. Houillier C, Lejeune J, Benouaich-Amiel A, Laigle-Donadey F, Criniere E, Mokhtari K, et al: Prognostic impact of molecular markers in a series of 220 primary glioblastomas. Cancer 106:2218-2223, 2006

20. Huse JT, Holland EC: Targeting brain cancer: advances in the molecular pathology of malignant glioma and medulloblastoma. Nat Rev Cancer 10:319-331, 2010

21. Ichimura K, Schmidt EE, Miyakawa A, Goike HM, Collins VP: Distinct patterns of deletion on 10p and 10q suggest involvement of multiple tumor suppressor genes in the development of astrocytic gliomas of different malignancy grades. Genes Chromosomes Cancer 22:9-15, 1998

22. Ichimura K, Vogazianou AP, Liu L, Pearson DM, Bäcklund LM, Plant K, et al: 1p36 is a preferential target of chromosome 1 deletions in astrocytic tumours and homozygously deleted in a subset of glioblastomas. Oncogene 27:2097-2108, 2008

23. Idoate MA, Echeveste J, Diez-Valle R, Lozano MD, Aristu J: Biological and clinical significance of the intratumour heterogeneity of PTEN protein expression and the corresponding molecular abnormalities of the PTEN gene in glioblastomas. Neuropathol Appl Neurobiol 40:736-746, 2014

24. Karsy M, Albert L, Murali R, Jhanwar-Uniyal M: The impact of arsenic trioxide and all-trans retinoic acid on p53 R273Hcodon mutant glioblastoma. Tumour Biol 35:4567-4580, 2014

25. Karsy M, Huang T, Kleinman G, Karpel-Massler G: Molecular, histopathological, and genomic variants of glioblastoma. Front Biosci (Landmark Ed) 19:1065-1087, 2014

26. Kita D, Yonekawa Y, Weller M, Ohgaki H: PIK3CA alterations in primary (de novo) and secondary glioblastomas. Acta Neuropathol 113:295-302, 2007

27. Koul D: PTEN signaling pathways in glioblastoma. Cancer Biol Ther 7:1321-1325, 2008

28. Krell D, Mulholland P, Frampton AE, Krell J, Stebbing J, Bardella C: IDH mutations in tumorigenesis and their potential role as novel therapeutic targets. Future Oncol 9:19231935, 2013

29. Labussière M, Boisselier B, Mokhtari K, Di Stefano AL, Rahimian A, Rossetto M, et al: Combined analysis of TERT, EGFR, and IDH status defines distinct prognostic glioblastoma classes. Neurology 83:1200-1206, 2014

30. Laxton RC, Popov S, Doey L, Jury A, Bhangoo R, Gullan R, et al: Primary glioblastoma with oligodendroglial differentiation has better clinical outcome but no difference in common biological markers compared with other types of glioblastoma. Neuro Oncol 15:1635-1643, 2013

31. Malkoun N, Chargari C, Forest F, Fotso MJ, Cartier L, Auberdiac P, et al: Prolonged temozolomide for treatment of glioblastoma: preliminary clinical results and prognostic value of p53 overexpression. J Neurooncol 106:127-133, 2012

32. Nakamura M, Watanabe T, Klangby U, Asker C, Wiman K, Yonekawa Y, et al: p14ARF deletion and methylation in genetic pathways to glioblastomas. Brain Pathol 11:159-168, 2001

33. Noushmehr H, Weisenberger DJ, Diefes K, Phillips HS, Pujara $\mathrm{K}$, Berman BP, et al: Identification of a $\mathrm{CpG}$ island methylator phenotype that defines a distinct subgroup of glioma. Cancer Cell 17:510-522, 2010

34. Ohgaki H, Kleihues P: Genetic profile of astrocytic and oligodendroglial gliomas. Brain Tumor Pathol 28:177-183, 2011
35. Ozawa T, Brennan CW, Wang L, Squatrito M, Sasayama T, Nakada M, et al: PDGFRA gene rearrangements are frequent genetic events in PDGFRA-amplified glioblastomas. Genes Dev 24:2205-2218, 2010

36. Parsons DW, Jones S, Zhang X, Lin JCH, Leary RJ, Angenendt $P$, et al: An integrated genomic analysis of human glioblastoma multiforme. Science 321:1807-1812, 2008

37. Philip R, Carrington L, Chan M: US FDA perspective on challenges in co-developing in vitro companion diagnostics and targeted cancer therapeutics. Bioanalysis 3:383-389, 2011

38. Phillips JJ, Aranda D, Ellison DW, Judkins AR, Croul SE, Brat DJ, et al: PDGFRA amplification is common in pediatric and adult high-grade astrocytomas and identifies a poor prognostic group in IDH1 mutant glioblastoma. Brain Pathol 23:565-573, 2013

39. Rich JN, Hans C, Jones B, Iversen ES, McLendon RE, Rasheed BKA, et al: Gene expression profiling and genetic markers in glioblastoma survival. Cancer Res 65:40514058, 2005

40. Ruano Y, Ribalta T, de Lope AR, Campos-Martín Y, Fiaño C, Pérez-Magán E, et al: Worse outcome in primary glioblastoma multiforme with concurrent epidermal growth factor receptor and p53 alteration. Am J Clin Pathol 131:257-263, 2009

41. Salkeni MA, Zarzour A, Ansay TY, McPherson CM, Warnick RE, Rixe O, et al: Detection of EGFRvIII mutant DNA in the peripheral blood of brain tumor patients. J Neurooncol 115:27-35, 2013

42. Sami A, Karsy M: Targeting the PI3K/AKT/mTOR signaling pathway in glioblastoma: novel therapeutic agents and advances in understanding. Tumour Biol 34:1991-2002, 2013

43. Schmidt MC, Antweiler S, Urban N, Mueller W, Kuklik A, Meyer-Puttlitz B, et al: Impact of genotype and morphology on the prognosis of glioblastoma. J Neuropathol Exp Neurol $61: 321-328,2002$

44. SongTao Q, Lei Y, Si G, YanQing D, HuiXia H, XueLin Z, et al: IDH mutations predict longer survival and response to temozolomide in secondary glioblastoma. Cancer Sci 103:269-273, 2012

45. Stancheva G, Goranova T, Laleva M, Kamenova M, Mitkova A, Velinov N, et al: IDH1/IDH2 but not TP53 mutations predict prognosis in Bulgarian glioblastoma patients. Biomed Res Int 2014:654727, 2014

46. Stupp R, Mason WP, van den Bent MJ, Weller M, Fisher B, Taphoorn MJB, et al: Radiotherapy plus concomitant and adjuvant temozolomide for glioblastoma. $\mathbf{N}$ Engl J Med 352:987-996, 2005

47. Taylor TE, Furnari FB, Cavenee WK: Targeting EGFR for treatment of glioblastoma: molecular basis to overcome resistance. Curr Cancer Drug Targets 12:197-209, 2012

48. Verhaak RGW, Hoadley KA, Purdom E, Wang V, Qi Y, Wilkerson MD, et al: Integrated genomic analysis identifies clinically relevant subtypes of glioblastoma characterized by abnormalities in PDGFRA, IDH1, EGFR, and NF1. Cancer Cell 17:98-110, 2010

49. Watanabe T, Vital A, Nobusawa S, Kleihues P, Ohgaki H: Selective acquisition of IDH1 R132C mutations in astrocytomas associated with Li-Fraumeni syndrome. Acta Neuropathol 117:653-656, 2009

50. Weller M, Kaulich K, Hentschel B, Felsberg J, Gramatzki D, Pietsch T, et al: Assessment and prognostic significance of the epidermal growth factor receptor vIII mutation in glioblastoma patients treated with concurrent and adjuvant temozolomide radiochemotherapy. Int J Cancer 134:2437-2447, 2014

51. Wemmert S, Ketter R, Rahnenführer J, Beerenwinkel N, Strowitzki M, Feiden W, et al: Patients with high-grade gliomas harboring deletions of chromosomes $9 p$ and $10 q$ benefit from temozolomide treatment. Neoplasia 7:883-893, 2005 
52. Wick W, Hartmann C, Engel C, Stoffels M, Felsberg J, Stockhammer F, et al: NOA-04 randomized phase III trial of sequential radiochemotherapy of anaplastic glioma with procarbazine, lomustine, and vincristine or temozolomide. J Clin Oncol 27:5874-5880, 2009

53. Wick W, Platten M, Meisner C, Felsberg J, Tabatabai G, Simon M, et al: Temozolomide chemotherapy alone versus radiotherapy alone for malignant astrocytoma in the elderly: the NOA-08 randomised, phase 3 trial. Lancet Oncol 13:707-715, 2012

54. Wong AJ, Ruppert JM, Bigner SH, Grzeschik CH, Humphrey PA, Bigner DS, et al: Structural alterations of the epidermal growth factor receptor gene in human gliomas. Proc Natl Acad Sci U S A 89:2965-2969, 1992

55. Zhao J, Ma W, Zhao H: Loss of heterozygosity $1 \mathrm{p} / 19 \mathrm{q}$ and survival in glioma: a meta-analysis. Neuro Oncol 16:103112,2014
56. Zheng H, Ying H, Yan H, Kimmelman AC, Hiller DJ, Chen AJ, et al: p53 and PTEN control neural and glioma stem/progenitor cell renewal and differentiation. Nature 455:11291133,2008

\section{Author Contributions}

Conception and design: Karsy, Neil. Analysis and interpretation of data: Neil. Drafting the article: Karsy, Neil. Critically revising the article: all authors. Reviewed submitted version of manuscript: all authors. Approved the final version of the manuscript on behalf of all authors: Karsy.

\section{Correspondence}

Michael Karsy, Department of Neurosurgery, The University of Utah, 175 N. Medical Dr. E, Salt Lake City, UT 84132. email: michael.karsy@hsc.utah.edu. 